\title{
Satellite Image Classification \\ Using a Divergence-Based Fuzzy c-Means Algorithm
}

\author{
Dong-Chul Park \\ Dept. of Electronics Engineering, Myong Ji University, Korea \\ parkd@mju.ac.kr
}

\begin{abstract}
A satellite image classifier scheme by using a Fuzzy c-Means $(\mathrm{FcM})$ algorithm is proposed in this paper. The $\mathrm{FcM}$ algorithm adopted in this paper is a Gradient-based FcM with Divergence measure $(\mathrm{GFcM}(\mathrm{D}))$ and it utilizes the Divergence measure to exploit the statistical nature of the image data and thereby improves the classification accuracy. Experiments and results on a set of satellite images demonstrate that the proposed GFcM(D)-based classifier outperforms conventional algorithms such as the traditional Self-Organizing Map (SOM) and Fuzzy c-Means (FcM) in terms of classification accuracy.
\end{abstract}

\section{Introduction}

Traditionally, conventional clustering algorithms such as the Self Organizing Map (SOM) 1] and the k-means algorithm [2 have seen the widest use in practice. However, they assign an object to a single class and ignore the possibility that the object may also belong to other classes. Fuzzy clustering techniques have also been proposed for clustering problems. One of the most widely used algorithms employing fuzzy clustering techniques is the Fuzzy c-Means (FcM) algorithm. The FcM algorithm was originally introduced by Bezdek in 1981 as an improvement on earlier clustering algorithms such as the SOM and the k-Means [3]-[5]. In the FcM, an object can belong to several classes at the same time but with different degrees of certainty, which are measured by the membership function. The FcM algorithm has more robust capabilities in comparison with the SOM and k-means and has been successfully applied to many clustering applications.

The Gradient-based Fuzzy c-Means (GFcM) algorithm introduced by Park 67. overcomes the drawback that each iteration requires the use of all the data at once. GFcM combines the characteristics of the SOM (presenting one datum at a time and applying the gradient descent method) and the FcM algorithm (continuous values of the membership grades in the range $[0,1])$. In the FcM algorithm, all the data are present in the objective function, and the gradients are set to zero in order to obtain the equations necessary for minimization. In contrast, only one datum at a time is required in the GFcM.

In this paper, a classification method for satellite image data by employing the GFcM algorithm with Divergence measure $(\mathrm{GFcM}(\mathrm{D}))$ [8, 9] is proposed. While 
the GFcM algorithm has been shown to give high clustering accuracy [6], it is also been demonstrated that the divergence measure can provide better modeling of statistic data such as image data. Therefore, this combination is expected to yield an improvement for image classification in terms of classification.

The remainder of this paper is organized as follows. Section 2 summarizes the Fuzzy c-Means and Gradient-based Fuzzy c-Means algorithms. Section 3 describes the Gradient-based Fuzzy c-Means algorithm with Divergence measure. Section 4 presents experiments and results on satellite image data sets including comparisons with other conventional algorithms. Conclusions are presented in Section 5.

\section{Gradient-Based Fuzzy c-Means(GFcM) Algorithm}

\subsection{Fuzzy c-Means(FcM) Algorithm}

Bezdek first generalized the fuzzy ISODATA by defining a family of objective functions $J_{m}, 1<m<\infty$, and established a convergence theorem for that family of objective functions 34. For FcM, the objective function is defined as :

$$
J_{m}(U, \boldsymbol{v})=\sum_{k=1}^{n} \sum_{i=1}^{c}\left(\mu_{k i}\right)^{m}\left(d_{i}\left(\boldsymbol{x}_{k}\right)\right)^{2}
$$

where $d_{i}\left(\boldsymbol{x}_{k}\right)$ denotes the distance from the input data $\boldsymbol{x}_{k}$ to $\boldsymbol{v}_{i}$, the center of the cluster $i, \mu_{k i}$ is the membership value of the data $\boldsymbol{x}_{k}$ to the cluster $i$, and $m$ is the weighting exponent, $m \in 1, \cdots, \infty$, while $n$ and $c$ are the number of input data and clusters, respectively. Note that the distance measure used in FcM is the Euclidean distance.

Bezdek defined a condition for minimizing the objective function with the following two equations [34]:

$$
\begin{aligned}
\mu_{k i} & =\frac{1}{\sum_{j=1}^{c}\left(\frac{d_{i}\left(\boldsymbol{x}_{k}\right)}{d_{j}\left(\boldsymbol{x}_{k}\right)}\right)^{\frac{2}{m-1}}} \\
\boldsymbol{v}_{i} & =\frac{\sum_{k=1}^{n}\left(\mu_{k i}\right)^{m} \boldsymbol{x}_{k}}{\sum_{k=1}^{n}\left(\mu_{k i}\right)^{m}}
\end{aligned}
$$

The FcM finds the optimal values of group centers iteratively by applying Eq. (2) and Eq. (3) in an alternating fashion.

\subsection{Gradient-Based Fuzzy c-Means(GFcM) Algorithm}

The FcM in Eq. (2) and Eq. (3) uses all data to update the center value of the cluster, but the GFcM that is used in this paper was developed to update the center value of the cluster with a given individual data sequentially [67]. Given one datum $\boldsymbol{x}_{k}$ and $c$ clusters with centers at $\boldsymbol{v}_{j},(j=1,2, \cdots, c)$, the objective function to be minimized is:

$$
J_{k}=\mu_{k 1}^{2}\left(\boldsymbol{v}_{1}-\boldsymbol{x}_{k}\right)^{2}+\mu_{k 2}^{2}\left(\boldsymbol{v}_{2}-\boldsymbol{x}_{k}\right)^{2}+\cdots+\mu_{k c}^{2}\left(\boldsymbol{v}_{c}-\boldsymbol{x}_{k}\right)^{2}
$$


with the following constraint:

$$
\mu_{k 1}+\mu_{k 2}+\cdots+\mu_{k c}=1
$$

The basic procedure of the gradient descent method is that starting from an initial center vector, $\boldsymbol{v}_{i}(0)$, the gradient $\Delta J_{k}$ of the current objective function can be computed. The next value of $\boldsymbol{v}_{i}$ is obtained by moving to the direction of the negative gradient along the error surface such that:

$$
\boldsymbol{v}_{i}(n+1)=\boldsymbol{v}_{i}(n)-\eta \frac{\partial J_{k}}{\partial \boldsymbol{v}_{i}(n)}
$$

where $n$ is the iteration index and

$$
\frac{\partial J_{k}}{\partial \boldsymbol{v}_{i}(n)}=2 \mu_{k i}^{2}\left(\boldsymbol{v}_{i}(n)-\boldsymbol{x}_{k}\right)
$$

Equivalently,

$$
\boldsymbol{v}_{i}(n+1)=\boldsymbol{v}_{i}(n)-2 \eta \mu_{k i}^{2}\left(\boldsymbol{v}_{i}(n)-\boldsymbol{x}_{k}\right)
$$

where $\eta$ is a learning constant.

A necessary condition for optimal positions of the centers for the groups can be found by the following:

$$
\frac{\partial J_{k}}{\partial \mu}=0
$$

After applying the condition of Eq. (7), the membership grades can be found as:

$$
\mu_{k i}=\frac{1}{\sum_{j=1}^{c}\left(\frac{d_{i}\left(\boldsymbol{x}_{k}\right)}{d_{j}\left(\boldsymbol{x}_{k}\right)}\right)^{2}}
$$

More detailed explanation about GFcM can be found in 677.

\section{GFcM with Divergence Measure}

In addition to the advantages of GFcM, GFcM was extended to another version that can deal with the probabilistic data. In the extended version, the selection of a proper distance measure between two data vectors should be extremely important since the performance of the algorithm largely depends on the distance measure to be adopted 4]. After evaluating various distance measures, the Divergence distance (Kullback-Leibler Divergence) between two Gaussian Probability Density Functions(GPDFs), $\boldsymbol{x}=\left(x_{i}^{\mu}, x_{i}^{\sigma^{2}}\right)$ and $\boldsymbol{v}=\left(v_{i}^{\mu}, v_{i}^{\sigma^{2}}\right), i=1, \cdots, d$, has been chosen as the distance measure in $\operatorname{GFcM}(\mathrm{D})$ [411]:

$$
\begin{aligned}
& D(\boldsymbol{x}, \boldsymbol{v}) \\
& =\sum_{i=1}^{d}\left(\frac{x_{i}^{\sigma^{2}}+\left(x_{i}^{\mu}-v_{i}^{\mu}\right)^{2}}{v_{i}^{\sigma^{2}}}+\frac{v_{i}^{\sigma^{2}}+\left(x_{i}^{\mu}-v_{i}^{\mu}\right)^{2}}{x_{i}^{\sigma^{2}}}-2\right)
\end{aligned}
$$




$$
=\sum_{i=1}^{d}\left(\frac{\left(x_{i}^{\sigma^{2}}-v_{i}^{\sigma^{2}}\right)^{2}}{x_{i}^{\sigma^{2}} v_{i}^{\sigma^{2}}}+\frac{\left(x_{i}^{\mu}-v_{i}^{\mu}\right)^{2}}{x_{i}^{\sigma^{2}}}+\frac{\left(x_{i}^{\mu}-v_{i}^{\mu}\right)^{2}}{v_{i}^{\sigma^{2}}}\right.
$$

where $x_{i}^{\mu}$ and $x_{i}^{\sigma^{2}}$ denote $\mu$ and $\sigma^{2}$ values of the $i^{t h}$ component of $\boldsymbol{x}$, respectively, while $v_{i}^{\mu}$ and $v_{i}^{\sigma^{2}}$ denote $\mu$ and $\sigma^{2}$ values of the $i^{\text {th }}$ component of $\boldsymbol{v}$, respectively.

The GFcM used in this paper is based on the FcM algorithm. However, instead of calculating the center parameters of the clusters after applying all the data vectors in the FcM, the GFcM updates their center parameters at every presentation of data vectors. By doing so, the GFcM can converge faster than the FcM [67. To deal with probabilistic data such as the GPDF, the GFcM(D) updates the center parameters, mean and variance, according to the distance measure shown in Eq. (9). That is, the membership grade for each data vector $\boldsymbol{x}$ to the cluster $i$ is calculated by the following equation:

$$
\mu_{i}(\boldsymbol{x})=\frac{1}{\sum_{j=1}^{c}\left(\frac{D\left(\boldsymbol{x}, \boldsymbol{v}_{i}\right)}{D\left(\boldsymbol{x}, \boldsymbol{v}_{j}\right)}\right)^{2}}
$$

After finding the proper membership grade from an input data vector $\boldsymbol{x}$ to each cluster $i$, the GFcM(D) updates the mean and variance of each center as follows:

$$
\begin{gathered}
\boldsymbol{v}_{i}^{\mu}(n+1)=\boldsymbol{v}_{i}^{\mu}(n)-\eta \mu_{i}^{2}(\boldsymbol{x})\left(\boldsymbol{v}_{i}^{\mu}(n)-\boldsymbol{x}^{\mu}\right) \\
\boldsymbol{v}_{i}^{\sigma^{2}}(n+1)=\frac{\sum_{k=1}^{N_{i}}\left(\boldsymbol{x}_{k, i}^{\sigma^{2}}(n)+\left(\boldsymbol{x}_{k, i}^{\mu}(n)-\boldsymbol{v}_{i}^{\mu}(n)\right)^{2}\right)}{N_{i}}
\end{gathered}
$$

where

$-\boldsymbol{v}_{i}^{\mu}(n)$ and $\boldsymbol{v}_{i}^{\sigma^{2}}(n)$ : the mean and variance of the cluster $i$ at the time of iteration $n$

- $\boldsymbol{x}_{k, i}^{\mu}(n)$ and $\boldsymbol{x}_{k, i}^{\sigma^{2}}(n)$ : the mean and variance of the $k^{t h}$ data in the cluster $i$ at the time of iteration $n$

- $\eta$ and $N_{i}$ : the learning gain and the number of data in the cluster $i$

GFcM(D) has been successfully applied to various data classification problems [8]- [10].

\section{Experiments and Results}

For the evaluation of the proposed image data classifier based on GFcM(D), satellite image data sets collected. The satellite image data set consists of different image classes (categories) in which each class contains different areas 15 15. 


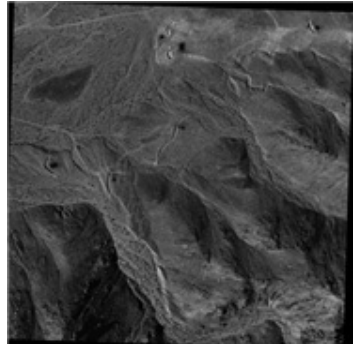

(a)

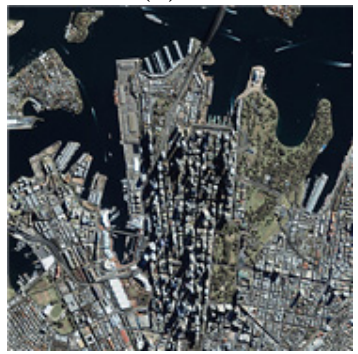

(d)

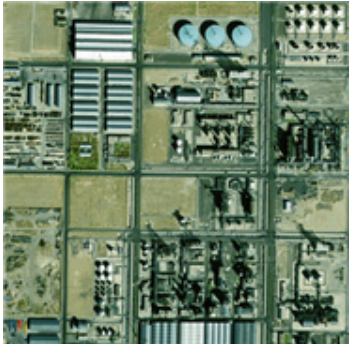

(b)

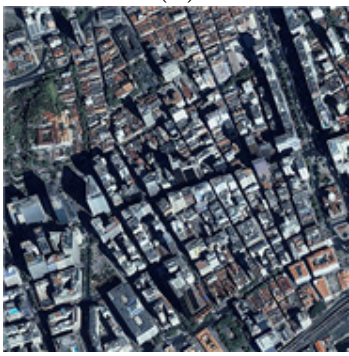

(e)

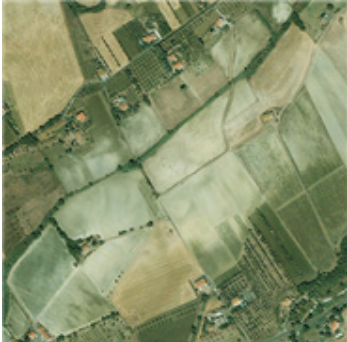

(c)

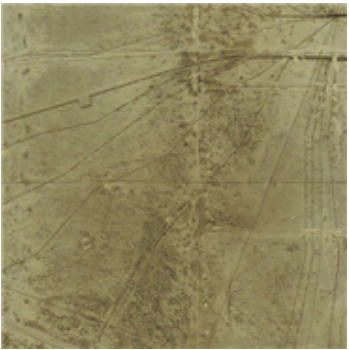

(f)

Fig. 1. Examples of satellite image data set:(a) Mountain area, (b) Factory area (c)Farm area, (d) Harbor area, (e) Urban area, and (f) Desert Area

Fig. 1 shows examples of mountain area, factory area, farming area, harbor area, urban area, and desert area. Each class consists of 100 images with different views resulting in a total of 600 images in the data set.

In order to obtain the texture information from the image, conventional texture descriptors based on a frequency domain analysis such as Gabor filters [12] and wavelet filters [13] are often used. However, these algorithms often induce a high computational load for feature extraction and are not suitable for real-time applications. In this paper, the Discrete Cosine Transform (DCT) is adopted for extracting the texture information from each block of the image [14. The DCT transforms the image from the spatial domain into the frequency domain.

For the localized representation, images are transformed into a collection of $8 \times$ 8 blocks. Each block is then shifted by an increment of 2 pixels horizontally and vertically. The DCT coefficients of each block are then computed and returned in 64 dimensional coefficients. Only the 32 lowest frequency DCT coefficients that are visible to the human eye are kept. Finally, a GPDF with a 32-dimensional mean vector and a $32 \times 32$ covariance matrix is used to represent the content of the image.

For each image class, the distribution of its feature vectors by a number of code vectors is calculated. Each code vector represents a group with its own mean and covariance matrix. During testing, the class of each image is decided by using a Bayesian classifier. To evaluate the proposed algorithm, its performance is compared with the performances of conventional algorithms such as SOM and 


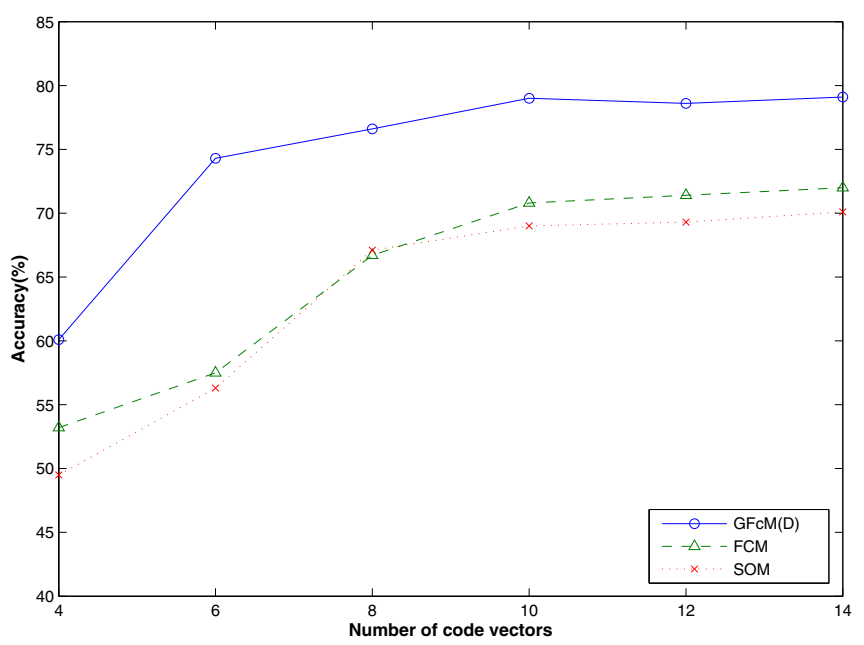

Fig. 2. Overall classification accuracies using different algorithms

Table 1. Classification accuracy (\%) of different algorithms using 12 code vectors

\begin{tabular}{|c|c|c|c|c|c|c|c|}
\hline & Mountain & Factory & Farm & Harbor & Urban & Desert & Overall \\
\hline \hline SOM & 58.3 & 67.1 & 66.3 & 73.2 & 81.5 & 69.2 & $\mathbf{6 9 . 3 \%}$ \\
\hline FcM & 63.6 & 70.2 & 65.7 & 75.6 & 83.2 & 70.0 & $\mathbf{7 1 . 4 \%}$ \\
\hline GFcM(D) & 70.3 & 72.5 & 70.4 & 85.6 & 90.4 & 82.1 & $\mathbf{7 8 . 6 \%}$ \\
\hline
\end{tabular}

FcM. Fig. 2] shows the performance in terms of the correct classification for three algorithms with several numbers of code vectors in a range from 4 to 14 . Table 1 shows the performance for each image class, using different algorithms with 12 code vectors. The classification performances for different algorithms are fairly saturated with 12 code vectors. Note that the SOM and the FcM algorithm do not use the covariance information. From the result shown in Fig. 2 and Table 1. we can infer that the algorithm that uses the covariance information, GFcM(D), usually outperforms the SOM and the FcM which use the Euclidean distance as their distance measure. The results also show that $\mathrm{GFcM}(\mathrm{D})$ provides far better accuracy over the other two algorithms.

\section{Conclusion}

In this paper, a new approach for classification of satellite images using a clustering algorithm is proposed. This paper shows how the mean and variance information of satellite image data are utilised using the Gradient-Based Fuzzy c-Means algorithm with Divergence measure. Experiments are successfully performed on a database with each image class such as mountain area, factory area, farm area, harbor area, urban area, and desert area. Based on the results of this 
experiment and previous experiments, it is expected that the GFcM(D) algorithm will have broad applicability. However, the classification results between mountain area and farm area or between urban area and factory area are far from satisfactory. More research on the selection of feature values in addition to the DCT values will help to discriminate some areas. Future work will include some standard evaluation protocols and baseline algorithms for the object recognition task in addition to FcM and SOM.

Acknowledgments. This work was supported by National Research Foundation of Korea Grant funded by the Korean Government (2010-0009655) and by the IT R\&D program of The MKE/KEIT (10040191, The development of Automotive Synchronous Ethernet combined IVN/OVN and Safety control system for 1 Gbps class).

\section{References}

1. Kohonen, T.: The Self-Organizing Map. Proc. of IEEE 78, 1464-1480 (1990)

2. Hartigan, J.: Clustering Algorithms. Wiley, New York (1975)

3. Bezdek, J.C.: A convergence theorem for the fuzzy ISODATA clustering algorithms. IEEE Trans. Pattern Anal. Mach. Intel., 1-8 (1980)

4. Bezdek, J.C.: Pattern Recognition with Fuzzy Objective Function Algorithms. Plenum, New York (1981)

5. Windham, M.P.: Cluster Validity for the Fuzzy cneans clustering algorithm. IEEE Trans. Pattern Anal. Mach. Intel., 357-363 (1982)

6. Park, D.C., Dagher, I.: Gradient Based Fuzzy c-Means Algorithm. In: Proc. of IEEE Int. Conf. on Neural Networks, pp. 1626-1631 (1994)

7. Looney, C.: Pattern Recognition Using Neural Networks, pp. 252-254. Oxford University Press, New York (1997)

8. Park, D.-C.: Classification of MPEG VBR Video Data Using Gradient-Based FCM with Divergence Measure. In: Wang, L., Jin, Y. (eds.) FSKD 2005. LNCS (LNAI), vol. 3613, pp. 475-483. Springer, Heidelberg (2005)

9. Park, D.-C., Nguyen, D.-H., Beack, S.-H., Park, S.: Classification of Audio Signals Using Gradient-Based Fuzzy c-Means Algorithm with Divergence Measure. In: Ho, Y.-S., Kim, H.-J. (eds.) PCM 2005. LNCS, vol. 3767, pp. 698-708. Springer, Heidelberg (2005)

10. Park, D.-C., Woo, D.-M.: Image Classification Using Gradient-Based Fuzzy cMeans with Divergence Measure. In: Proc. of IJCNN, pp. 2520-2524 (2008)

11. Fukunaga, K.: Introduction to Statistical Pattern Recognition, 2nd edn. Academic Press Inc. (1990)

12. Daugman, J.G.: Complete Discrete 2D Gabor Transform by Neural Networks for Image Analysis and Compression. IEEE Trans. Acoust., Speech, and Signal Processing 36, 1169-1179 (1988)

13. Pun, C.M., Lee, M.C.: Extraction of Shift Invariant Wavelet Features for Classification of Images with Different Sizes. IEEE Trans. Pattern Anal. Mach. Intel. 26(9), 1228-1233 (2004)

14. Huang, Y.L., Chang, R.F.: Texture Features for DCT-Coded Image Retrieval and Classification. In: Proc. of IEEE ICASSP, vol. 6, pp. 3013-3016 (1999)

15. Park, D.-C.: Classification of Satellite Images Using Partitioned Feature-based Classifier Model. In: Proc. of ICISA (2011)

16. Park, D.-C., Jeong, T., Lee, Y., Min, S.-Y.: Satellite Image Classification Using a Classifier Integration Model. In: Proc. of AICCSA, pp. 90-94 (2011) 